Voix et Images

\title{
La spiritualité des Jésuites de la Nouvelle-France : L'interprétation des Visions de Jean de Brébeuf (1640)
}

\section{Guy Laflèche}

Volume 11, numéro 3 (33), printemps 1986

Yolande Villemaire

URI : https://id.erudit.org/iderudit/200581ar

DOI : https://doi.org/10.7202/200581ar

Aller au sommaire du numéro

Éditeur(s)

Université du Québec à Montréal

ISSN

0318-9201 (imprimé)

1705-933X (numérique)

Découvrir la revue

Citer cet article

Laflèche, G. (1986). La spiritualité des Jésuites de la Nouvelle-France :

L'interprétation des Visions de Jean de Brébeuf (1640). Voix et Images, 11(3),

464-474. https://doi.org/10.7202/200581ar d'utilisation que vous pouvez consulter en ligne. 


\title{
La spiritualité des Jésuites de la Nouvelle-France: L'interprétation des Visions de Jean de Brébeuf (1640)
}

\author{
par Guy Laflèche, Université de Montréal
}

Durant un siècle (1860-1960), le catholicisme d'État a imposé au Canada français une vision messianique de son histoire: son passé, c'était Une épopée mystique, les Origines religieuses du Canada ${ }^{1}$, et son avenir, le ciel, pour avoir accepté la mission de convertir l'Amérique au catholicisme et à la civilisation française. Cette histoire nationale, mise au point par l'élite cléricale, se présentait comme une suite de tableaux héroïques (le Découvreur, le Fondateur, le Missionnaire, le Sauvage, le Coureur des bois, l'Explorateur et pour finir l'Habitant et son Curé) où l'épisode des «Saints Martyrs canadiens" occupait la position centrale et constitue la clé de l'épopée: un "groupe" de huit Jésuites (six missionnaires et deux domestiques), qui auraient été «martyrisés» par les Indiens, avait scellé dans le sang la mission de la Nation. Ce mythe des Saints Martyrs canadiens, enseigné jusqu'à tout récemment dans les écoles avec un luxe remarquable de détails sadomasochistes, a toujours un impact considérable sur l'imaginaire québécois ${ }^{2}$.

La figure dominante des Saints Martyrs est Jean de Brébeuf (1593-1649). Arrivé en Nouvelle-France en 1625, il va passer trois ans chez les Hurons où les Récollets avaient ouvert une mission. En 1629, la colonie est conquise par les Anglais et Brébeuf rentre en France. De retour en 1633, il sera nommé supérieur de la mission huronne et on peut dire que sa biographie s'identifie dès lors à l'histoire de cette mission jésuite où il trouvera la mort en 1649 . Ce sera aussi la fin de la mission. Depuis 1647, les Iroquois d'Onontagué envahissent la Huronie par vagues successives, détruisant les villages et assimilant leurs habitants. Le 16 mars 1649, Jean de Brébeuf et Gabriel Lalemant (jeune missionnaire depuis moins d'un an dans la colonie) sont capturés par l'armée iroquoise qui attaque par surprise le village de Saint-Louis; blessés mortellement au cours du combat, ils sont suppliciés et mis à mort. Ils seront les seuls missionnaires de Nouvelle-France à mourir victimes du supplice archaïque des Amérindiens et Gabriel Lalemant aura été le seul à en subir les trois phases, car Jean de Brébeuf meurt après trois heures d'un supplice qui en dure normalement près de vingt-quatre.

C'est à cause de sa mort et non de sa vie que Jean de Brébeuf est la figure dominante des Saints Martyrs (contrairement à Isaac Jogues, assassiné d'un coup de hache, on connaît les terribles détails de son supplice). L'hagiographie et la biographie édifiante nous le présentent sous deux aspects antithétiques propres à mettre son portrait en valeur: d'un côté le géant, le lion 
ou plutôt le «bœuf" (comme le porte son nom, a-t-on l'habitude de dire), de l'autre le mystique, le visionnaire ou le prophète. On se doute bien que la seconde caractéristique est incomparablement plus importante que la première. Toutefois, avant son martyre, personne ne s'est jamais douté qu'il s'agissait de rien de moins que d'un "mystique», un homme doué d'une spiritualité extraordinaire, sujet d'extases, de rêves prophétiques et de visions, victime d'apparitions diaboliques et récipiendaire de grâces et faveurs célestes incroyables... Bien au contraire, les deux Relations et les quelques lettres ${ }^{3}$ que nous avons de lui nous présentent un rude travailleur tout entier à sa tâche apostolique, d'une spiritualité certes bien rigoriste, pessimiste et mortificatrice (c'est la "spiritualité de la Croix" très significative des missionnaires de la Huronie ${ }^{4}$ ), mais tout ce qu'il y a de plus régulière. Ses biographes auraient dû être formidablement surpris à la découverte de ce que l'on appelle aujourd'hui son "Journal spirituel", s'ils n'avaient justement choisi de remplacer sa biographie par le portrait d'un mystique.

En effet, tout ce que l'on dit ${ }^{5}$, encore aujourd 'hui, de Jean de Brébeuf sort tout cru du portrait qu'en a tracé Paul Ragueneau au chapitre 5 de sa Relation de 1649 , tout de suite après son récit circonstancié du martyre ${ }^{6}$. Voici la conclusion, le thème ou la thèse que Ragueneau place en tête de son portrait de Brébeuf:

Souuent Nostre Seigneur s'est apparu à luy, quelquefois en estat de gloire, mais d'ordinaire portant sa Croix, ou bien y estant attaché; qui imprimoit dedans son cour des desirs si ardens de beaucoup souffrir pour son nom, que quoy qu'il eut beaucoup souffert en mille occasions, des peines, des fatigues, des persecutions, des douleurs; tout ne luy estoit rien: (il) se plaignoit de son malheur, croyant que iamais il n'auoit rien souffert, \& que Dieu ne le trounoit pas digne de luy faire porter la moindre partie de sa Croix.

Nostre Dame luy est aussi tres-souuent apparuë, qui d'ordinaire laissoit en son ame des desirs de souffrir, mais auec des douceurs si grandes, \& vne telle soufmission aux volontez de Dieu, qu'en suite son esprit en demeuroit dans vne paix profonde, \& dans vn sentiment esleué des grandeurs de Dieu. l'espace de plusieurs iours ${ }^{7}$.

Une vingtaine d'années plus tard, dans sa Vie de la Mère Catherine de Saint-Augustin ${ }^{8}$, ce sont précisément ces deux alinéas qu'il reproduira pour résumer la biographie de Brébeuf, ajoutant encore aux apparitions et ravissements la pénétration de conscience et le don de prophétie:

Saint Joseph, les Anges \& plusieurs Saints du Paradis, s'estoient souvent fait voir à luy; souvent Dieu luy avoit donné la grace de penetrer dans le fond des cours, \& d'y voir l'état interieur des consciences, ou pour les dons de Dieu qui y étoient répandus, ou pour les pechez les plus cachez, \& que qui que ce soit au monde ne pouvoit sçavoir. 
Nôtre Seigneur luy avoit revelé tres-souvent les choses futures: \& quelquefois il luy faisoit connoitre les bontez toutes particulieres \& toutes amoureuses qu'il avoit pour ceux qui s'abondonnoient eux-mémes, \& tous leurs interêts, pour le salut des Ames: \& luy avoit fait voir la place, qu'il leur reservoit dans le Ciel pour une éternité 9 .

Bref, voilà un portrait de Jean de Brébeuf entièrement dominé par l'extraordinaire, c'est-à-dire par les manifestations sensibles des puissances de l'au-delà sur sa personne, ce que ses biographes ont nommé son "mysticisme" ${ }^{10}$.

Or ce portrait contredit absolument tout ce que nous savons de Jean de Brébeuf par ailleurs, soit à travers ses écrits et ce qu'on a toujours dit sur lui de son vivant, soit par l'intermédiaire des faits et des événements de sa biographie qui caractérisent son caractère et sa pensée. Est-ce à dire que le portrait "extraordinaire» que nous en fait Paul Ragueneau ne s'appuie sur aucun document ou témoignage? Pour les témoignages, il n'y en a qu'un, celui de l'illuminé Pierre Chaumonot qui fantasme les miracles avec une telle naïveté qu'il fallait toute l'aveugle amitié de Ragueneau pour lui faire confiance "l. Il faut dire, à sa décharge, que celui-ci avait trouvé un document de première main qui le confirmait et le confortait dans une interprétation de la mort de son confrère où il projetait son propre désir et sa propre crainte $\mathrm{du}$ martyre.

Voici comment Paul Ragueneau présente le document en question après avoir donné le thème de son portrait dans les deux alinéas reproduits plus haut:

Il auoit eu commandement d'escrire ces choses extraordinaires, qui se passoient en luy, au moins celles dont il pourroit plus aisément se ressouuenir, car elles estoient trop frequentes, \& le soin du salut du prochain, à peine luy donnoit-il quelque loisir d'écrire de fois à autre. Voicy les deux dernieres choses que i'ay trouuées dans ses memoires ${ }^{12}$.

Comme on le verra, les mémoires en questions ne sont en réalité qu'un seul et unique document que Paul Ragueneau éditera lui-même quelques années plus tard dans un recueil, le Manuscrit de 1652, où il nous est parvenu sous le titre «Quelques graces, visions, illustrations \& autres remarques extraites des manuscrits du P. Jean de Brebeuf "13. Les deux citations qui suivent sont en effet les «deux dernières choses» qu'on trouve dans ce document, c'est-à-dire ses deux derniers fragments:

Quantité de croix me sont apparuës, que i'embrassois toutes tresvolontiers. La nuit suivante estant en oraison, me conformant aux volontez de Dieu sur moy, \& luy disant, Fiat voluntas tua ("Que ta volonté soit faite», Matthieu, 6:10), Domine, quid me vis facere? ("Seigneur, que veux-tu que je fasse?", Actes, 6: 10), i'ay entendu vne voix qui m'a dit, Tolle, Lege ("Prends et lis", Augustin, les Confessions, 8: 12). Le iour estant venu, i'ay pris en 
main le petit liure de l'Imitation de Iesus-Christ, \& sans dessein ie suis tombé sur le chapitre De regia via sanctae crucis ( De la voie royale de la sainte croix", chapitre qui se situe très exactement au centre de l'Imitation). Depuis ce temps-là, i'ay senty dans mon ame vne grande paix, \& vn repos dans les occasions de souffrir.

Sur le soir estant en oraison deuant le tres-saint Sacrement, i'ay veu en esprit sur mes habits, \& sur les habits de tous nos Peres, sans qu'aucun en fust excepté, des taches toutes de sang, ce qui m'a laissé dans vn sentiment d'admiration ${ }^{14}$.

Mais il faut préciser que Paul Ragueneau ajoute ensuite à ces visions tirées du manuscrit de Brébeuf l'interprétation suivante:

Nous n'en sçauons pas dauantage, \& si peut-estre Dieu n'a point voulu nous aduertir, \& par ces croix, \& ce sang, qu'il nous fera la mesme grace, dont il a voulu recompenser les merites de ce bon Pere, de mourir pour son nom, \& de répandre nostre sang pour l'establissement de sa gloire ${ }^{15}$.

En réalité, Ragueneau en savait bien davantage, car il avait sous les yeux l'interprétation de Brébeuf lui-même qui contredisait absolument celle qu'il propose implicitement à son lecteur dévot ici, celle qui fait voir dans ces taches de sang l'image de la mort martyre dont Brébeuf aurait eu dans cette vision la prescience. Voici plutôt le texte complet de Brébeuf:

Octavo octobris annj 1644, in sacello B. Mariae apud hurones, sero dum proces fierent, ante coenam, mihi sum videre, quasj maculas quasdam sanguineas aut violaceas in vestibus omnium nostrorum et meis etiam, ita ut nemo exciperetur; cum autem id admirarer venit mihi in mentem omnes justitiae nostrae quasj pannus menstruatus (Isaias, 64:6) ${ }^{16}$.

Comme on le voit, en particulier au contexte de la citation biblique, cette vision n'avait absolument rien à voir avec le martyre. Toutefois, même s'il doit tronquer sa citation pour en contredire le sens, Ragueneau ne se trompe pas tout à fait, ni avec lui son lecteur: d'abord, comme on l'a vu, il projette sa crainte et son désir du martyre dans celui de Brébeuf (et son interprétation le dit ici explicitement ); ensuite, rapportant une vision et une vision présupposée prophétique, il peut s'estimer mieux placé que Brébeuf lui-même pour l'interpréter. La question qui se pose est plutôt de savoir comment les biographes et historiens modernes ont pu reconduire sans sourciller ce portrait extravagant d'un visionnaire, d'un prophète ou d'un «mystique». Comment? En prenant soin de ne jamais s'interroger sur la nature des textes qu'ils citaient à titre de "documents historiques", comme s'il suffisait que les documents en question datent du XVII ${ }^{\mathrm{e}}$ siècle et qu'ils soient authentiques pour qu'ils aient le bon sens qu'on leur donne. En réalité, on vient de voir que la "biographie» de Paul Raguenau, qui sert de modèle à toutes les autres, est un portrait édifiant qui transforme et interprète les textes au lieu de les citer; or on va voir maintenant que ce portrait édifiant d'un "visionnaire» repose tout entier 
sur l'interprétation fautive d'un document qu'on a présenté comme des extraits d'un «Journal spirituel» qui n'a jamais existé.

Des «extraits", c'est ce que propose en effet le titre du document dans le Manuscrit de 1652 et c'est à quoi ressemble assez cette suite de 39 fragments ${ }^{17}$. Mais dès la première lecture, on voit bien qu'il ne s'agit nullement d'un recueil ou d'une anthologie de textes (extraits ou citations), mais bel et bien d'une suite de notes (morceaux ou fragments) dont l'unité saute aux yeux. Quels en sont les caractères généraux? On constate d'abord que les 39 fragments sont précisément datés et qu'à trois exceptions près (et bien faciles à expliquer, puisque ces exceptions consignent justement des dates «mémorables ": fragments 6 à 8) tous les fragments sont dans un ordre rigoureusement chronologique. Ils se répartissent de janvier 1630 à octobre 1644 , mais ne se distribuent pas régulièrement, puisque 24 des 39 fragments sont de 1640 . Or ces 24 fragments (à l'exception de la note extatique du fragment 28 ) consignent tous des visions.

De façon plus générale, les 39 fragments se classent entre six genres qui se succèdent à peu près chronologiquement : (a) il s'agit d'abord de résolutions ou de conclusions qui ferment des retraites ou des examens de conscience ( 1 à 4,10 ); (b) on trouve ensuite la consignation des dates mémorables dont il vient d'être question (6 à 8); et (c) on trouve aussi les textes de deux vœux (9 et 12). Tout cela forme comme une sorte de préambule, qui a pu être consigné rétrospectivement et dont le véritable objet suit - les manifestations de l'extraordinaire: (d) la consignation de deux extases ( 5 et 28 , sans compter les visions qui se transforment en extase ou débouchent sur elle, comme au fragment 29). (e) la description des 25 visions et (f) finalement le récit de deux rêves prophétiques de janvier et février 1641 ( 37 et 38).

La première et meilleure preuve que ces fragments n'ont jamais été découpés ni probablement choisis, c'est qu'ils ont toujours la même structure, celle par exemple de la vision des vêtements souillés ${ }^{18}$ : (1) Brébeuf situe précisément la vision: date, situation géographique et circonstances biographiques ou historiques; (2) il décrit ensuite la vision sous la forme d'un tableau, d'une suite de tableaux ou parfois d'un bref récit: dans ces descriptions, l'effort de précision est remarquable; (3) très souvent, Brébeuf complète cette description déjà méticuleuse par des renseignements techniques et spécialisés sur la nature de la manifestation: on sait, contrairement à ce qu'écrit Ragueneau non sans mauvaise foi, que Brébeuf n'est l'objet d'aucune apparition, mais d'extase, de rêveș et de visions, dont il précise scrupuleusement la forme (distincte ou pas, vue dans le sommeil ou par les yeux de l'esprit, etc.) et la durée; à ce sujet, il précise encore les sentiments qu'il a éprouvés à cette occasion s'ils ne sont pas évidents ou s'ils contredisent le sens commun; (4) enfin, c'est la conclusion du fragment, Brébeuf expose l'interprétation qu'il a alors donnée à la vision ou précise qu'elle était inexpliquée. Ce dernier trait est particulièrement remarquable: jamais il n'analyse, n'interprète ou ne commente de quelque façon le contenu d'aucune de ces manifestations extraordinaires. Il les décrit et les consigne froidement, objectivement et minutieusement, non pas pour les faire partager, ni même pour en garder la trace 
sous forme de mémorial, mais tout simplement parce qu'on lui a demandé de consigner ses visions par écrit et qu'il se soumet au jugement de son lecteur. Il ne s'agit aucunement d'un texte spirituel, herméneutique et prosélytique, ni même d'un document hermétique, et encore moins mystique: il s'agit tout simplement d'un texte juridique.

En effet, c'est de Jérôme Lalemant, supérieur de la mission huronne de 1638 à 1644, que Brébeuf auoit eu commandement d'ecrire ces choses extraordinaires, qui se passoient en luy ${ }^{19}$. L'ordre du supérieur ne peut dater que de la retraite que Brébeuf fait à Sainte-Marie du 11 au 18 février 1640 (les fragments 13 à 18 consignent ses visions au cours de cette retraite). À partir de cette date, le mémoire (car c'est bien de cela qu'il s'agit) consigne les visions durant un an, jusqu'au 7 février 1641 (fragment 38, l'avant-dernier). Du printemps 1641 au 7 septembre 1644, Brébeuf est à Québec, loin de l'autorité de Lalemant, où il n'a plus à consigner de visions. Or c'est précisément de l'automne où Brébeuf revient en Huronie et du même automne où Jérôme Lalemant est déchargé du supériorat de cette mission (bien qu'il y reste encore jusqu'au printemps suivant) que date la dernière vision (le fragment 39 , du 8 octobre 1644). Bref, Jérôme Lalemant est celui par qui les visions arrivent...

Car il ne faudrait pas s'y tromper. Les supérieurs de la Compagnie de Jésus en Europe se sont très souvent inquiétés des comportements particuliers ou extraordinaires de ceux dont ils avaient la responsabilité. Comme leur fondateur Ignace de Loyola, ils ne craignent pas moins les "nouvelles spiritualités» et toute forme de "mysticisme" que le simple comportement un peu trop exalté de quelques-uns de leurs novices. Pourtant, c'est exactement le contraire qui se produit ici. Jérôme Lalemant, qui voit le diable et la Providence partout, manifeste une spiritualité maladivement orientée vers les manifestations sensibles des Toutes-Puissances: c'est à lui que nous devons tous les écrits excentriques des Jésuites de la Nouvelle-France (et en particulier de ceux dont il a écrit le martyre - Jogues, notamment); enfin, c'est lui qui découvrira le seul sorcier qu'on trouvera jamais dans la colonie, Daniel Vuil, et qui organisera avec l'évêque François de Laval son procès pour hérésie et son exécution; et par la même occasion, c'est toujours lui (et toujours avec François de Laval) qui aliènera cette pauvre Catherine de Saint-Augustin, ce cas d'obsession caractérisée se déclarant au moment où il lui confiera la garde de la jeune Barbe Hallay que Daniel Vuil aurait ensorcelée. Jérôme Lalemant est donc bien celui par qui les visions arrivent.

Mais, dira-t-on, les visions de Jean de Brébeuf, il ne les a tout de même pas inventées? Mais oui, puisqu'il les a montées en épingle, qu'il en a exigé un mémoire et qu'il les a donc prises au "sérieux" ou au pied de la lettre. Car le mémoire en question est justement là pour montrer que Jean de Brébeuf n'accordait à ses visions ni ce statut, ni cette importance, et il est clair qu'elles n'avaient pour lui rien d'extraordinaire (aux sens courant et spirituel du mot). Alors, de quoi s'agissait-il?

La réponse à cette question se trouve, me semble-t-il, à mi-chemin entre les réactions contraires des deux derniers commentateurs de ces visions: 
René Latourelle qui s'inquiète du caractère illuminé de Brébeuf et qui fait tout pour réduire l'importance de ses visions; François Roustang qui, au contraire, accorde toute son attention à ces visions et en fait la clé de sa personnalité mystique. Même s'il analyse minutieusement la part de Dieu et celle du Diable dans ces visions, René Latourelle se défend mal d'un certain malaise, puisqu'il croit devoir insister sur le fait que Brébeuf n'est pas un illuminé. Les visions ne symbolisent pour lui qu'un stade avancé ou très avancé de l'évolution mystique ${ }^{20}$, ce qui lui permet de laisser dans l'ombre la prémisse, à savoir que Jean de Brébeuf avait des visions et qu'il était donc un illuminé au sens le plus strict. Et c'est justement ce qu'entend démontrer François Roustang. À partir de la «bizarrerie» de son journal, il nous présente un Brébeuf assailli par les démons ${ }^{21}$; Dieu, écrit-il

permet qu'il soit investi comme une place forte par les puissances infernales. Elles prennent un jour le visage d'un Espagnol; une autre fois encore, c'est une vieille femme qui vient l'embrasser. Pendant des années (sic), Brébeuf mène le grand combat contre les forces invisibles qui dominent l'humanité22.

Ce délire fantasmagorique est bien entendu sans rapport avec les démons de Jean de Brébeuf: il s'agit plutôt de ceux de François Roustang qui n'a pas su appliquer la méthode jésuite du "discernement des esprits».

Voilà bien l'origine des démons de Jean de Brébeuf: les Exercices spirituels $^{23} \mathrm{~d}$ 'Ignace de Loyola. D'abord, Brébeuf consigne ses fragments à partir de sa retraite de janvier 1630 et ses visions à partir de celle de février 1640 ; par ailleurs, ses visions sont consignées pour la plupart à l'issue de ses exercices spirituels ou de dévotions qui s'y rattachent. Ensuite et surtout, les visions de Jean de Brébeuf ont été programmées par les exercices jésuites. La forme de l'objectif des Exercices spirituels est le "discernement des esprits" par l'analyse des «motions». En termes simples, on peut dire que les exercices ont pour but concret de prendre une décision (quelle qu'elle soit) qui doit être celle de Dieu et indifférente à toute chose créée. Pour cela, le retraitant doit savoir appliquer rigoureusement les règles (313-336) qui lui permettront de juger de l'origine de ses pensées ou des motions qu'il trouve en lui; car ces pensées, il peut les trouver en lui-même, mais elles peuvent aussi lui venir des puissances sensibles (182), c'est-à-dire des bons ou mauvais esprits. Or la méthode qui permet d'arriver, à travers ce discernement, à la décision que l'on doit prendre consiste en une série d'opérations réglées de compositions, d'applications et de colloques. La composition consiste à voir par le regard de l'imagination $(47)^{24}$ des tableaux concrets et à travers eux les choses invisibles; l'application des sens est l'effort d'imaginer la matérialité de cette composition par le regard, l'oreille, l'odorat, le goût et le toucher, par exemple en baisant les lieux où les personnages de la composition passent (120-126); et la contemplation se termine par un colloque où l'on doit réellement parler à Jésus, la Vierge, etc., comme on parle à son ami(54). Réellement? Si la pratique des exercices peut en définitive, dans une certaine mesure, être raisonnable, il est certain qu'ils n'assignent aucune limite à l'imagination et qu'ils tendent au contraire à faire apparaître les esprits. Bref, non seulement la méthode ne 
distingue jamais les compositions des visions, mais elle tend au contraire à passer des premières aux secondes... avant d'en venir aux apparitions. Il n'y a plus alors qu'à appliquer la méthode à elle-même pour que la question ne soit plus de distinguer les compositions des visions, mais de savoir si elles (les motions. comme les compositions devenues visions) viennent de Dieu ou si elles ne sont pas diaboliques, ce qui débouche sur le scrupule et l'obsession.

Voilà donc ce qui, à mes yeux, explique les visions de Jean de Brébeuf et en décrit la nature. Au cours de ses exercices spirituels, au temps de ses retraites et parfois même dans ses oraisons, les compositions prescrites par Ignace de Loyola se transforment en visions, c'est-à-dire que Jean de Brébeuf perd le sentiment de les provoquer ou de les "composer". Par conséquent, leur sens (symbolique) ne repose plus sur une idée préalable et consciente, mais sur une signification (inconsciente) que Brébeuf doit attribuer à la vision elle-même. Par conséquent, encore, la vision présente un caractère de réalité qui tient simplement au fait qu'elle n'est pas volontaire: voilà simplement une composition parfaitement réussie. Ce délire n'a absolument rien de caractérisé: d'une part ces visions sont nettement localisées (en cours d'oraison et particulièrement en retraite) et d'autre part elles n'impliquent aucun effet apparent sur son comportement. Bref, Jean de Brébeuf rêve et rêvasse, comme tous les hommes, et il croit fermement en l'existence de Dieu, du Diable, des anges et autres esprits. De ce point de vue, il n'a absolument rien d'un "visionnaire" et la meilleure preuve en est, encore une fois, ce mémoire constitué d'une suite de ces visions... qu'il distinguait donc soigneusement de la «réalité» (ce qu'un visionnaire bien entendu n'aurait jamais fait).

L'interprétation des visions de Jean de Brébeuf ne semblerait pas complète si nous ne prenions pas leur contenu en considération. Pourtant, la caractéristique la plus évidente de ce contenu est bien de n'avoir à peu près rien de remarquable, sinon justement de confirmer l'orientation profondément rigoriste (à commencer par l'immense dégoût de lui-même ${ }^{25}$, comme le dit si justement François Roustang) de sa spiritualité. À première vue, le corpus de ses visions ne permet pas de les classer par sujets. On constate tout au plus que huit fragments impliquent des objets symboliques, quatre présentent la Vierge et quatre autres des animaux. Le démon, que François Roustang voit partout, ne se trouve que dans quatre fragments et dans deux de ceux-ci ( 19 et 27) le démon ne se trouve impliqué que dans la signification de la vision; autrement, le diable prend la forme d'un lion (15) et une foule de démons apparaît sous la forme de chevaux à crin de bouc (11); et Brébeuf prend la peine d'indiquer qu'il n'a éprouvé aucune crainte dans les deux cas. Dans l'ensemble, d'ailleurs, les visions sont plutôt favorables et bénéfiques, comme le confirme le ravissement ou l'extase sur lequel elles débouchent souvent. Par ailleurs, un trait de leur contenu confirme nettement leur origine psycho-sociologique comme "compositions": la Vierge est décrite comme on la représente $(26,33)$, le diable a une figure de lion semblable à celle que l'on représente sur l'image du P. Joseph Anchieta (15), le Jésuite est tel que nos Pères sont peints ordinairement (23).... de sorte que de proche en proche, on se rend compte que la description, au centre de chacune des visions, est au 
sens strict un tableau, c'est-à-dire une composition, comme on l'a vu, jusque dans son contraire où le corps du Christ en croix est composé sous les traits (profondément bibliques) du lépreux - et non de la manière dont il est représenté ordinairement $(16)^{26}$.

Le Christ en croix, la Croix, est le thème qui s'impose avec le plus d'insistance, comme l'a bien vu René Latourelle. Quatre fragments (13, 14, 16 et 38 ) y sont explicitement consacrés, et le dernier d'entre eux, comme on l'a vu ${ }^{27}$, contient la référence au chapitre central de l'Imitation de Jésus-Christ sur ce thème de la Croix qui est en quelque sorte le sujet de cet ouvrage: l'abnégation de soi-même et la constance à souffrir toutes sortes d'adversités pour l'amour de Jésus-Christ ${ }^{28}$. Or l'essentiel est que ni ce thème, ni la spiritualité qu'il exprime, n'appartiennent au mémoire de Jean de Brébeuf sur ses visions, non plus qu'à ces visions: il s'agit du thème central de l'œuvre du missionnaire, comme de la spiritualité qu'il partage avec la plupart des Jésuites de Huronie ${ }^{29}$. C'est la spiritualité de la Croix $^{30}$, celle qui prend sur la difficile réalité de l'apostolat missionnaire la revanche sans éclat du renoncement, de l'amour des souffrances et de la joie des échecs. Un mépris souvent sordide de la vie, mais dont l'envers inattendu peut être parfois cette indifférence paisible qui fait le ravissement des visions de Jean de Brébeuf. Évasion - bien plus qu'élévation -. l'espace d'un Miserere, comme dit le fragment 11.

On voit maintenant quel contresens il y avait à faire des "visions» de Jean de Brébeuf des apparitions miraculeuses et autres phénomènes extraordinaires, quel non-sens il y avait à faire d'un mémoire juridique des extraits d'un Journal spirituel! Car c'était confondre la spiritualité profondément rigoriste de Brébeuf, éloignée de toute "mystiquerie», avec la spiritualité triomphaliste de ses supérieurs et biographes, cette spiritualité exaltée à la recherche du miraculeux et de l'extraordinaire. Le miracle ici est bien que l'on ait pu durant si longtemps s'interdire d'interpréter les visions de Jean de Brébeuf pour assurer le sens mythique de l'épisode des Saints Martyrs canadiens. L'objectif n’a jamais été, il est vrai, d'étudier la spiritualité des Jésuites de la Nouvelle-France. Il était plus simplement de faire.du catholicisme la religion de l'Êtat. Aussi a-t-on fait de ce Brébeuf-là, comme des Saints Martyrs canadiens qu'il symbolise, le patron du Canada français.

1. Selon le titre de l'ouvrage célèbre de Georges Goyau (Paris. Grasset, 1924).

2. Mon étude du mythe se trouvera au premier des cinq volumes de mon édition des textes narratifs sur l'épisode à paraitre sous le titre les Saints Martyrs canadiens et dont la publication devrait commencer au cours de l'hiver 1985-1986 (voir notamment le chap. 5 du vol. 1: "Histoire d'une cause: le culte des saints Martyrs canadiens"). Qu'il suffise de dire ici qu'aucun de ces Jésuites n'a jamais été "martyrisé" par les Amérindiens: quatre d'entre eux ont été suppliciés comme captifs de guerre et deux seulement (qui avaient été mortellement blessés au combat) en sont morts; les autres ont été assassinés ou tués au cours de combats et chacun dans des circonstances particulières. C'est l'hagiographie jésuite qui les a regroupés et en a fait des martyres. 
3. Il s'agit des Relations de la Huronie de 1635 et de 1636 publiées à la suite des Relations de la Nouvelle-France des mêmes années (éd. originale: Paris, Cramoisy, 1636 et 1637; édition diplomatique dans la collection de R.G. Thwaites, The Jesuit Relations.... Cleveland, Burrows Brothers, 1896-1901, 73 volumes, respectivement vol. 8 , p. 68-154 et vol. 10, p. 6-316, ce que je désignerai dorénavant comme suit: JR, 8: 68-154 et 10: 6-316). Et pour la correspondance, elle ne contient pas plus d'une douzaine de lettres de caractère essentiellement administratif (voir la bibliographie établie par René Latourelle en tête de son Étude sur les écrits de Jean de Brébeuf, Montréal, Immaculée-Conception, 2 vol., 1952-1953, 1: 11-20).

4. Je reviendrai plus bas sur cette spiritualité commune aux missionnaires de NouvelleFrance qui vivent, à l'écart des villages de la colonie française, de difficiles expériences d'acculturation. Voir ma caractérisation des spiritualités de la Croix, de la Crosse et du Crucifix chez les missionnaires Jésuites de Nouvelle-France en tête de mon édition de la Vie du Père Paul Ragueneau de Jacques Bigot, Montréal (VLB Éditeur, 1979. p. 29-34).

5. Ce sont ses biographies: celles de Félix Martin (1877), Paul Piron (1943), Joseph Robine (1949), F.X. Talbot (1949), René Latourelle (1952) et Joseph Donnelly (1975). L'ouvrage de René Latourelle (cité n. précédente) n'est pas à proprement parler une biographie, mais une lecture biographique de ses écrits. Tous ces biographes sont des Jésuites qui, se projetant dans la Vie de Brébeuf, n'avaient aucune raison de ne pas reconduire la biographie édifiante de Raguenau, même s'ils le font au terme d'un cheminement méthodologique de plus en plus "scientifique».

6. JR. 34: 158-164 (chapitre 11 de mon édition citée n. 1). En réalité il existe une version antérieure de ce portrait dans la lettre que Ragueneau adresse en latin au général Vincent Carafa en date du premier mars 1649 (Archives Générales de la Compagnie de Jésus, Rome, Gal. 109. doc. 125, deux copies, dont une autographe).

7. JR, 34: 160-162.

8. Paris. Florentin Lambert, 1671.

9. Réédition des Hospitalières de Québec, 1923, réimpression de 1977, p. 114.

10. "Brébeuf mystique et martyr", conclut René Latourelle au dernier chapitre de son étude citée n. 3. "Mystique appelé aux plus hautes faveurs», commence François Roustang en tête de son portrait spirituel (Jésuites de la Nouvelle-France, Paris, Desclée de Brouwer, coll. "Christus», 1961, p. 98). Peu importe ici la confusion entre le mysticisme et l'illuminisme, car les historiens des Jésuites de la Nouvelle-France ignorent tout de leurs spiritualités, trop occupés de la "vérité historique" des "martyres"...

11. Ragueneau invoque trois fois le témoignage (anonyme) de Chaumonot dans ce chapitre 5 de sa Relation de 1649: au sujet de confidences de Brébeuf faites quelque dix ans plus tôt et interprétées au lendemain de sa mort... Pour se convaincre du caractère puérilement illuminé de Chaumonot, il suffit de lire sa dévote autobiographie (éd. de J.-G. Shea à New York, Nouvelle Presse Cramoisy, 1858; et éd. de F. Martin à Paris, Oudin, 1885).

12. JR, 34: 162 .

13. Manuscrit des Archives de la Compagnie de Jésus du Canada français (Saint-Jérôme), document no 202, 302 p., cité ici dans l'édition diplomatique du tapuscrit d'Arthur Melançon, 1936 (la seule édition publiée est celle, souvent fautive, du Rapport de l'archiviste de la Province de Québec pour 1924-1925. Le titre du document de Jean dẹ Brébeuf qui nous occupera ici se trouve à la page 220 du manuscrit; le texte (latin entremêlé de quelques fragments français), p. 224-240, se trouve surmonté de la note suivante, signée Paul Ragueneau: "Quae sequuntur ex manuscriptis P. Joannis de Brebeuf excerpta sunt» ("Ce qui suit est extrait des manuscrits du P. Jean de Brébeuf"). Nous possédons deux éditions commentées du document: d'abord l'édition et la traduction de René Latourelle (ouvr. cité n. 3, 2: 202-237) et la traduction de 
François Roustang (ouvr. cité n. 10, p. 126- 138), et dans les deux cas le texte est présenté comme une collection d' "extraits» d'un "Journal spirituel» de Brébeuf, ce que croient d'ailleurs tous ses biographes.

14. JR, 34: 162. Les parenthèses sont de moi.

15. Ibid.

16. Manuscrit de 1652 , cité n. 13,p. 240; édition de R. Latourelle, citée n. 3, p. 209. Le 8 octobre 1644, dans la chapelle de Sainte-Marie des Hurons, pendant la prière du soir, avant le repas, il me sembla voir comme des taches de sang ou des traces violettes sur les vêtements de tous les nôtres et les miens aussi, et nul n'en était exempt; comme cela me plongeait dans l'étonnement, il me vint à l'esprit que toutes nos justices sont comme du linge souillé(traduction et référence de F. Roustang, cité n. 10, p. 137). Le contexte de la citation biblique est le suivant: et facti summus ut inmundus omnes nos / quasi pannus menstruatae universae iustitiae nostrae, nous avons tous été comme l'homme impur (le lépreux) et nos actions justes ont été comme un linge souillé (par les menstrues).

17. Pour le titre (voir la n. 13), il s'agit d'une authentification où "extraits" et "excerpta sunt» doivent d'abord s'entendre au sens de "tirés de». En ce qui concerne les 39 fragments auxquels je renverrai par leur numéro d'ordre, il faut les compter sur le manuscrit ou dans l'édition de R. Latourelle (citée n. 3), car F. Roustang y a ajouté (entre crochets) des "extraits» souvent hétérogènes de la Relation de 1649 et il a reporté à la toute fin le fragment 12 , du 18 août (1638), à cause d'une erreur de chronologie. On obtient 39 fragments en numérotant tous les alinéas du document (même s'ils se rapportent à la même journée ou peuvent former une unité), sauf pour le fragment 12 constitué du texte d'un vœu et de ses règles.

18. Fragment 39, reproduit plus haut (voir la n. 16).

19. JR, 34: 162 , déjà cité plus haut.

20. Ouvrage cité n. 3, vol. 2: Brébeuf n'est pas un illuminé (p. 183 et 219) et ses visions représentent un stade avancé (ou "très avancé», p. 222, n. 1) de l'évolution mystique (p. 183).

21. Ouvrage cité n. 10, p. 102.

22. Ibid., p. 99.

23. L'édition courante, en français, est aujourd'hui la traduction de François Courel (Paris, Desclée de Brouwer, coll. "Christus", 1960). C'est aux numéros des paragraphes que renverront les chiffres entre parenthèses dans la suite de mon exposé: on pourra donc s'y reporter dans n'importe quelle édition.

24. Brébeuf emploie la même formule: les yeux de l'esprit (33), en une vision intellectuelle ou imaginative (11), etc.

25. François Roustang, ouvr. cité n. 10, p. 99.

26. Toutes ces citations sont prises de la traduction de François Roustang (ouvr. cité n. 10).

27. Voir plus haut le texte qui figure en regard de la $\mathbf{n}$. 14. Et c'est bien ce que disait Paul Ragueneau lui-même, comme on le voit au texte cité en regard de la n. 7 .

28. L'Imitation de Jésus-Christ, 23:4, trad. Lamennais, Paris, Seuil, coll. "Points» 1961, p. 48.

29. Voir de ce point de vue mon analyse des deux Relations de Brébeuf dans aLe narrateur des Premières Relations ", Revue de l'Université d'Ottawa, 48: 1-2 (janvier 1978), p. 46-61.

30. Voir plus haut la n. 4. 